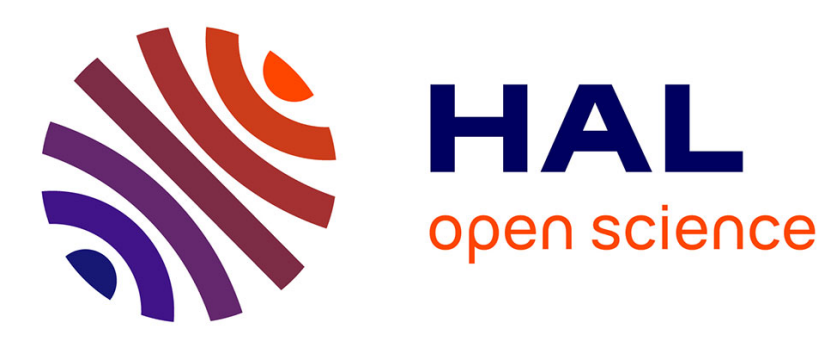

\title{
Visualisation of Physical Lung Simulation: an Interactive Application to Assist Physicians
}

Pierre-Frédéric Villard, Gabriel Fournier, Michaël Beuve, Behzad Shariat

\section{To cite this version:}

Pierre-Frédéric Villard, Gabriel Fournier, Michaël Beuve, Behzad Shariat. Visualisation of Physical Lung Simulation: an Interactive Application to Assist Physicians. Information Visualisation, 2006, London, United Kingdom. pp.65-70. hal-00849201

\section{HAL Id: hal-00849201 https://hal.inria.fr/hal-00849201}

Submitted on $30 \mathrm{Jul} 2013$

HAL is a multi-disciplinary open access archive for the deposit and dissemination of scientific research documents, whether they are published or not. The documents may come from teaching and research institutions in France or abroad, or from public or private research centers.
L'archive ouverte pluridisciplinaire HAL, est destinée au dépôt et à la diffusion de documents scientifiques de niveau recherche, publiés ou non, émanant des établissements d'enseignement et de recherche français ou étrangers, des laboratoires publics ou privés. 


\title{
Visualisation of Physical Lung Simulation: an Interactive Application to Assist Physicians
}

\author{
Pierre-Frédéric Villard, Gabriel Fournier, Michaël Beuve, Behzad Shariat \\ LIRIS, bat Nautibus, 8 bd Niels Bohr, 69622 Villeurbanne CEDEX, FRANCE \\ pierre-frederic.villard@liris.univ-lyon1.fr
}

\begin{abstract}
Radiation therapy of cancer necessitates accurate tumour targeting. Unfortunately, during the treatment the tumour and the related organs can undergo large displacement and deformation. Physicians need an estimation of these movements, for an adapted therapy. In this paper, we propose a methodology to provide physicians with reconstructed $4 D(3 D+$ time $) C T$ scans, considered as essential data. Moreover we propose an interactive visualisation tool, permitting the exploration of reconstructed $4 D$ CT scans as well as the generation of new CT scan sections in any direction of the $3 D$ space.
\end{abstract}

Keywords_Finite Element Method, Dynamic Deformable Model, Convolution, 4D CT scan, GPU

\section{Introduction}

In classical techniques for planning and evaluation of radiation therapy of cancer, patients are considered as 3D unmoving and unchanging objects resulting in a inadequate treatment and side effects. However, an efficient therapy requires to target precisely the treatment volumes by considering the patient as a $4 \mathrm{D}$ object, undergoing movements and deformations.

In our previous works, We studied lung motion using Finite Element techniques. For this the lungs volume were meshed at their initial state, then we calculated the evolution of the initial meshes. The obtained results from geometrical point of view are interesting, however the clinicians need that these geometrical data be converted into 4D-CT scan. This paper deals with this latter need.

Here, after a short recall of our approach to compute mesh deformation, we present our methodology of 4D-CT simulation. This is performed in two steps: First, the evolution of matter density of each finite element cell due to its deformation is computed. Next, the matter density of each cell is transformed into the Hounsfield density. The final CT scan cell density is an average of Hounsfield density function over the cell.

Moreover, We present a rendering tool permitting the user to visualise dynamically the $4 \mathrm{D}$ reconstructed
CT scans, and to observe lung slices with any arbitrary position and orientation that can be interactively chosen. For this, the computer graphics techniques: geometry proxy and texture streaming in conjunction with graphic cards facilities are used.

\section{4D CT scan}

Static CT scan images of lung are not reliable because of breathing. Time-dependant tumour displacement must be explicitly provided. A 4D CT scan, which is a set of 3D CT scan indexed by time, is more appropriate.

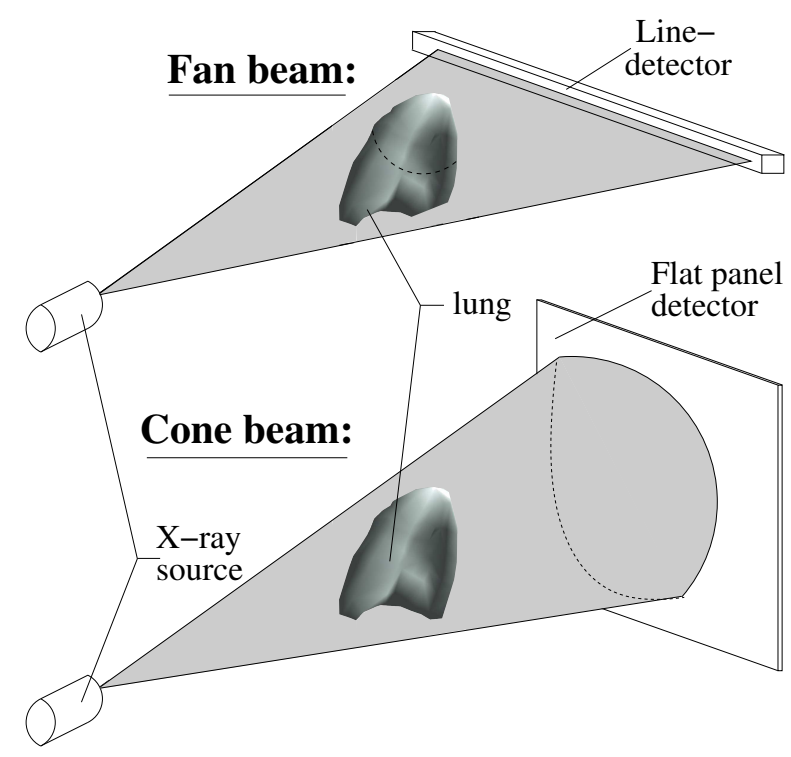

Figure 1: Tomography device: fan beam and cone beam

Practically, one can obtain 4D CT scan using adapted tomodensitometric device like fan beam or cone beam. Fan beam is a flat beam used in the well known sequential CT or in spiral CT. Then it provides 1D information. Cone beam tomography instead consists of a conical beam and provides 2D information at a time (Cf Figure 1).

Extension to 4D CT scan is appreciably similar in both cases: while the patient breathes normally, his respiratory 
cycle as well as CT scan slices are extracted. This coincidence provides with an indexing of CT scan slices on the respiratory cycle (Cf Figure 2). However a difference between the two devices remains: in the case of fan beam, slice indexing is performed after the reconstruction phase [5] whereas in the case of cone beam, 3D construction is carried out after the time indexing phase [12]. The advantage of these protocols is to obtain rather quickly, approximately 4 minutes, a complete and accurate 3D tomography with a clear reduction of breathing artefacts [11].

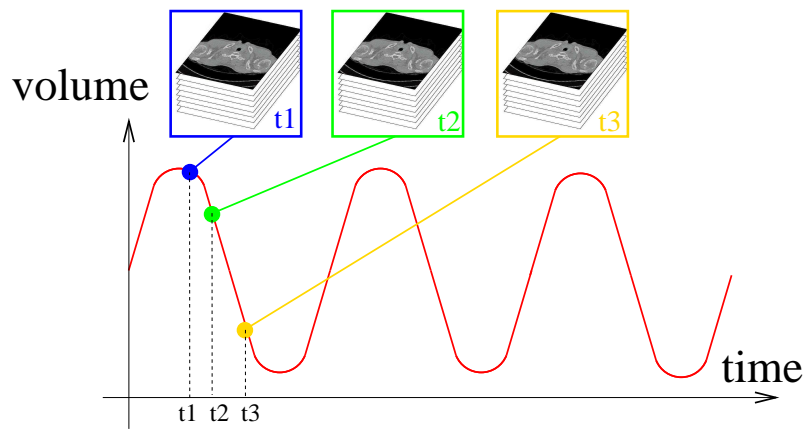

Figure 2: Various 3D CT scans indexed on a breathing curve

Models to simulate organ motion, such as biomechanical approaches, are however still relevant for many aspects. 1- It can predict evolution of tumour in shape and in position at any time. In the case of 4D CT scan images, segmentation has to be performed on all the slices and for each time. 2- In principle, the 4D CT scan is valid only the day of acquisition and for the configuration of breathing. Modelling can predict motions in any situation even if the patient modify his breathing (thoracic or diaphragmatic). 3- Simulation could provide a great number of intermediate states and 4- There will be no dose problem for patient due to unnecessary acquisitions.

\section{Physical lung simulation}

We have presented in [13] a method to simulate pulmonary motions with a technique based on continuous mechanics.

The geometrical and mechanical data are customised to each patient and provided by our medical partners: Léon Bérard centre and Louis Pradel Hospital, both in Lyon (France). Lung geometry is extracted from the segmentation of CT scan section and is modelled by a 3D mesh adapted to the resolution of non-linear mechanical problems. Presently, we assume that the lung is homogeneous, isotropic and that its constitutive equation is well characterised by Poisson's ratio and Young modulus. This latter is extracted from a physiological measurement: the compliance [10].

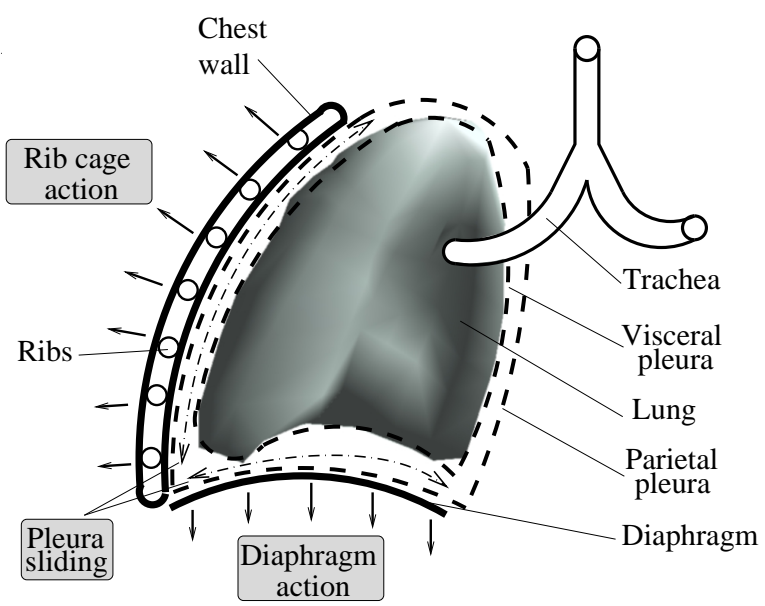

Figure 3: Lung boundary conditions based on real anatomy

For boundary conditions, lung is fixed at the trachea and is subject to an homogeneous negative pressure, without any additional stress in accordance with the role of pleura [4], as indicated by the anatomists (Cf Figure 3).

The resolution of the system is carried out by a standard finite element software and the result is a displacement field for the whole lung mesh nodes.

This information should now be transformed into CT scan slices for physicians use.

\section{4D CT scan generation}

Our aim is to calculate the matter density within each mesh element in order to produce a 4D CT scan using scanner convolution parameters in direct accordance with the clinical scanner device. An example of such conversion is displayed on Figure 4. We aim at combining the lung model with the remapped density values from an initial CT scan to provide a dynamic estimate of feature changes within the model lung as it deforms.

\section{1 interpolation data}

As a first assumption, we could admit that the density is constant inside a mesh element. Nevertheless, this is too crude and inconsistent with continuous mechanics formalism, which considers each function continuous. Moreover, we simulate motion with finite element method, using then interpolation functions for each element $E_{i}$. So, we will keep here the same philosophy.

A shape function for each finite element describes geometry and topology. The expression of a function $f(\mathbf{P})$ at each point $\mathbf{P}$ is a linear combination of interpolation function $N_{j}$ and function values $f\left(\mathbf{P}_{\mathbf{j}}\right)$, where $j$ indexes the nodes: 


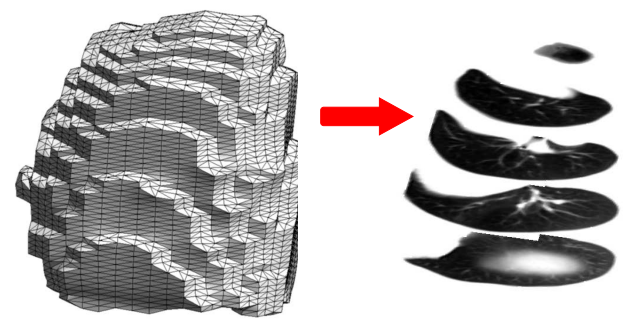

Figure 4: Lung CT scan conversion

$$
f(\mathbf{P})=\sum_{j \in E_{i}} N_{j}(\mathbf{P}) f\left(\mathbf{P}_{\mathbf{j}}\right)
$$

\section{2 matter density extraction}

The main quantity calculated with finite element simulation is generally displacement field. It has to be converted into density to simulate CT scan. We propose to use the mass conservation equation (Equation.2) because matter density $\rho_{M}\left[K g \cdot m^{-3}\right]$ is related to velocity $\mathbf{V}$, that is displacement after time integration.

$$
\frac{\partial \rho_{M}}{\partial t}+\operatorname{div}\left(\rho_{M} \cdot \mathbf{V}\right)=0
$$

Knowing the displacement over a short time step, one can calculate the variation $\Delta_{\rho}$ of density. An iterative process starting from a set of initial matter density extracted from just one CT scan transforms displacement into timedependant density.

Practically, it consists in inserting in equation (2) the discretised function expression previously seen in $\S 4.1$ for the displacement $\mathbf{U}$ and the matter density. The density variation in time for any point $P$ in element $E_{i}$ reads:

$$
\Delta \rho \approx \sum_{j, j^{\prime}} \rho\left(\mathbf{P}_{j}\right) \operatorname{div}\left(N_{j}(\mathbf{P}) N_{j^{\prime}}(\mathbf{P}) \mathbf{U}_{i}\left(\mathbf{P}_{j^{\prime}}\right)\right)
$$

Finally equation (3), provided the time step is small, allows us to calculate the $\Delta \rho$ from the simple knowledge of node position, node densities at previous state and node displacements over the time step.

The iterative process may induce cumulated error due to the approximation in the time integration of density. We checked the mass conservation to perform a quantitative evaluation of our method to compute the time dependant matter density function. The error (Cf Figure 5) between the initial mass (computed from the initial segmented CT scan) and the final mass (computed by integrating the matter density over the final deformed lung volume) leads to a mass loss error inferior to $0.2 \%$ (average curve on the figure). Besides, we have computed the minimum and the maximum mass loss errors over all the mesh elements and for all the inflating levels. The maximum error on an element is always inferior to $1 \%$.

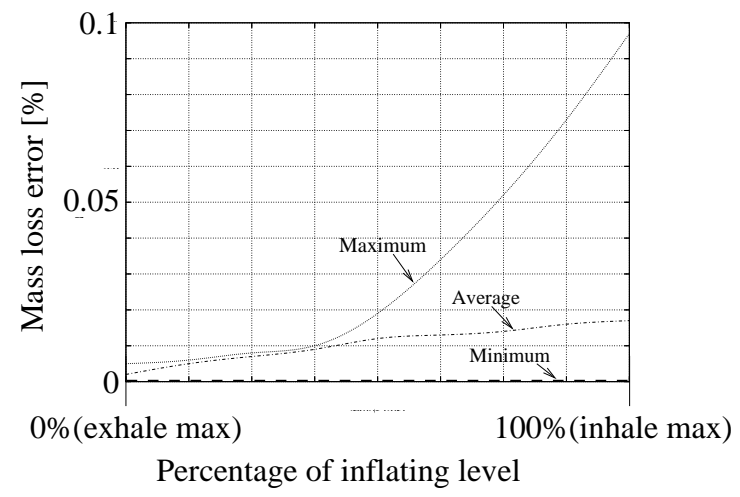

Figure 5: Mass loss error evolution with time

This control reveals the actual degree of reliability of our approach. The continuous matter density has now to be converted into Hounsfield density for each CT scan voxel $V_{l}$.

\subsection{CT scan convolution}

First, the matter density $\rho_{M}(\mathbf{P})$ at a solid point inside a mesh element $\mathbf{P}$-previously computed- is linked with the Hounsfield value at this point, $\rho_{H}(\mathbf{P})$, by an affine function:

$$
\rho_{H}(\mathbf{P})=a \rho_{M}(\mathbf{P})+b
$$

where coefficients $a$ and $b$ have to be extracted from a calibration of the clinical device [9]. Obviously the coeficients $a$ and $b$ implicitly include the relation between matter density and electronic density.

This Hounsfield density of solid points inside a finite element has now to be taken into account for each voxel. Figure 6 pictures a comparison between CT scan and Finite element geometry.

The density function will be expressed as an interpolation forms of node value $\rho_{H}\left(\mathbf{P}_{\mathbf{j}}\right)$ (4.1). To take globally into account the operations related to the CT scan device and image processing, we convolute the density by a filter function $f_{\sigma}$, where $\sigma$ represents the experimental standard deviation:

$$
\rho_{H}\left(\mathbf{P}^{\prime}\right)=f_{\sigma} \otimes \rho_{H}(\mathbf{P})
$$

The final CT scan voxel density $D_{H}\left(V_{l}\right)$ is supposed to be an average of Hounsfield density function over the voxel and, if $P^{\prime}$ denotes a point inside a CT scan voxel, it reads:

$$
D_{H}\left(V_{l}\right)=\frac{1}{\mathcal{V}_{l}} \int_{V_{l}} \rho_{H}\left(\mathbf{P}^{\prime}\right) d \mathbf{P}^{\prime 3}
$$




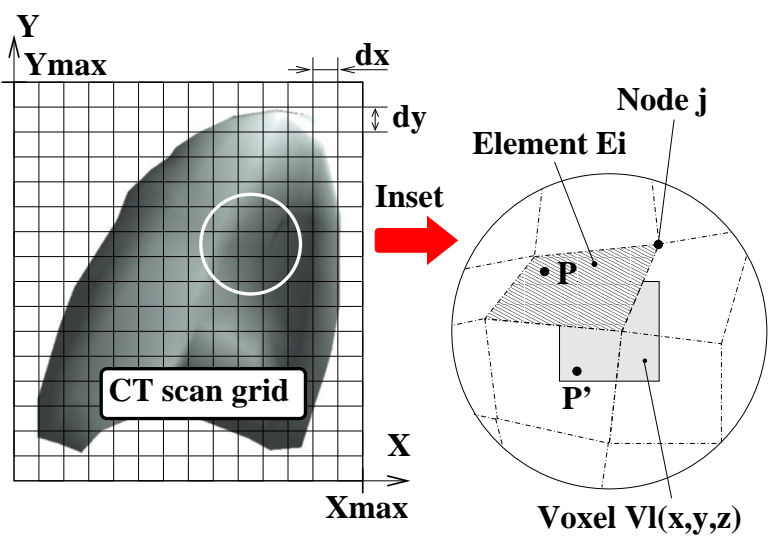

Figure 6: Geometrical parameter for CT scan convolution

Injecting Equation.5 in Equation.6, we obtain:

$$
\begin{aligned}
& D_{H}\left(V_{l}\right)=\frac{a}{\mathcal{V}_{l}} \\
& \sum_{E_{i}} \int_{E_{i}} \rho_{M}(\mathbf{P}) \int_{V_{l}} f_{\sigma}\left(\left\|\mathbf{P}-\mathbf{P}^{\prime}\right\|^{2}\right) d \mathbf{P}^{\prime 3} \cdot d \mathbf{P}^{3}+b
\end{aligned}
$$

The latter simplification comes from the fact that the space integral of $f_{\sigma}$ is equal to 1 . The expression $\int_{V_{l}} f_{\text {sigma }}\left(\left\|\mathbf{P}-\mathbf{P}^{\prime}\right\|^{2}\right) d \mathbf{P}^{\prime 3}$ is independent of the function $\rho_{h}(\mathbf{P})$ and since the geometry of $V_{l}$ is known and constant, an analytical expression can be derived. The function $f_{\sigma}$ is chosen as a 3D Gaussian function to be integrated over the field given by the intersection of a voxel with a sphere.

This full algorithm generates a 4D CT scan from the output of our biomechanical model. 4D CT scan consists of a mass of data for which we propose a tool for a convenient usual exploration by physician and medical physicist.

\section{Interactive visualisation of reconstructed lung CT scans}

We present here a visualisation tool for time-dependant CT scans.

Current programmable graphics hardware now have the processing power required to handle large amount of data, like reconstructed animated 3D lung CT scans, which can reach up to 335MB for 10 time steps (Cf Equation (8)).

$$
\begin{aligned}
& X_{\max } \cdot Y_{\max } \cdot Z_{\text {max }} \cdot N_{\text {time }} . \text { sizeof }(\text { float }) \\
& =256 \times 256 \times 128 \times 10 \times 4 \mathbf{B} \\
& =335 \mathrm{MB}
\end{aligned}
$$

CT scan can be represented by a 3D array of floats. Each value represents the density of the tissue inside a voxel. To externally visualise lungs, we need to extract the isosurface defined by the material density of lung. Many works have been done to exploit programmable hardware for volume visualisation. Extraction of a triangulated representation of an isosurface is a possible approach [8] that can be implemented on a GPU [6]. As, in addition to its surface, we also want to visualize the 'inside' of the lung (through cuts in the surface), we choose an other approach that does not require transformation of the volumetric data into a triangle set. This other approach is based on ray tracing. Rays are sent from the viewer into the volume, and their intersections with the isosurface are computed by sampling the volume along them [7]. This approach can be implemented in software but can also benefit from hardware acceleration [1].

The volumetric data we want to render are stored within a 3D texture arrays which will be sampled by the graphics hardware within a fragment program that takes advantage of parallel processing capabilities of the GPU. The sampling of the volume is performed by rendering a geometry proxy like multiple planes orthogonal to the viewing direction. For each pixel on screen of each plane (rectangular quad), the volume is sampled to know whether the sampling point is inside or outside the isosurface. Pixels obtained for external points are discarded; points inside the surface are rendered. Such a naive algorithm allows to visualise the volume, but discontinuities due to discreet sampling leads to visible 'woodcut' artefacts when the surface is shaded. A better estimation of the isosurface position needs to be computed (Cf Figure 7).

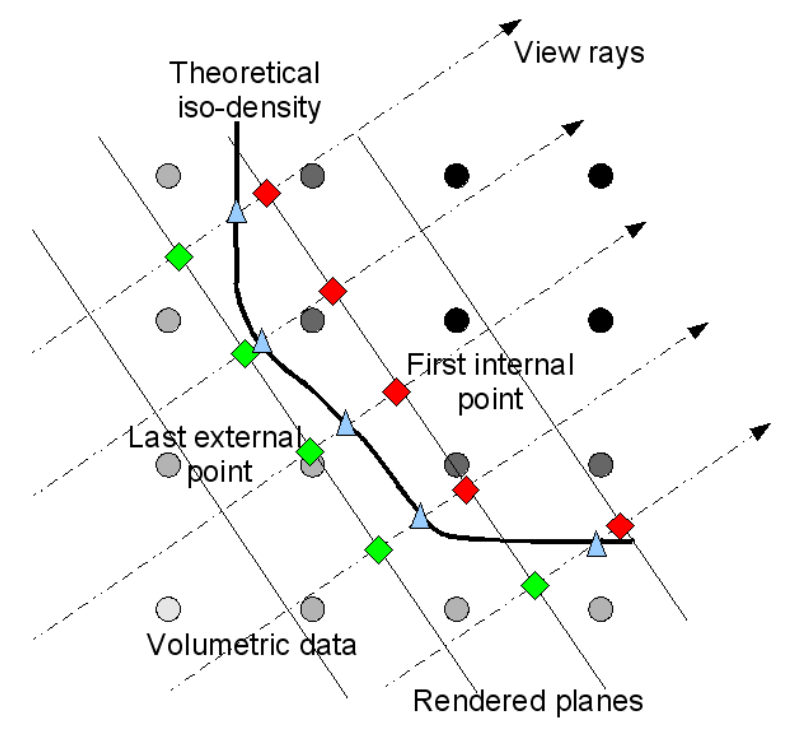

Figure 7: Isosurface estimation

When a point is found inside the isosurface, the volume is sampled again along the ray in the direction of the viewer to find the last point outside the isosurface. The position of 
the isosurface is linearly interpolated between the two sampled points. Nicer interpolations can be used [3] but they require more processing power and eventually more texture accesses. Once the isosurface position estimated with no discontinuity, it is possible to shade the surface. Shading computations require knowledge of the normal to the surface. Normals can be pre-computed using the 3D gradient of the volume and stored in another 3D texture array. Interpolating the position of the isosurface and shading the pixels requires a few texture accesses that limit the frame rate. To speed up the rendering, we use the deferred shading approach, in a first pass only the first internal point is computed for each pixel and its position is written to an off screen framebuffer. In a second rendering pass, the visible points computed during the first pass are used, first to interpolate the isosurface position, and then to shaded this surface. This way, fewer texture accesses and computations are made as only the visible points are shaded. Visualising the outside of the lung is interesting but seeing inside the lung is more important for cancer treatment.

Some volume visualisation algorithms allow to render multiple segmented isosurface with transparency [2]. We preferred to display none segmented data inside the lung to avoid deleting important information for the user. To see 'inside' the lung, we do not sample the volume in front of a cut plane. Pixels on the first rendered plane are directly given by the density value of the volume at their 3D position of the intersection point. These density values are tone mapped interactively according to the user's desire to hide or reveal certain features. This approach allows the user to interactively visualise CT scans the way he is used to, with the added ability to see lung slices with any arbitrary position and orientation that can be interactively chosen (Cf Figure 8).

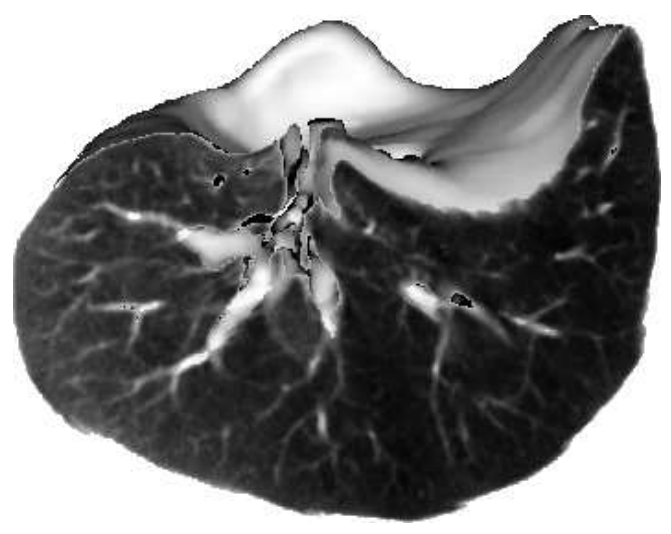

Figure 8: Rendering of a slice into lung

The main goal of our work is to visualise the reconstructed CT scan that shows the lung motion and defor- mation. The main difference with regards to the previously described algorithm is to perform 4D interpolations instead of 3D interpolation. A 3D texture is used for each reconstructed volume for each 'key frame'; linear interpolations are made between two 3D textures. To keep animation smooth, the interpolation parameters and the two textures depend on the elapsed time between the two successive rendered frames. The main difficulty is that the generated data can be too voluminous to fit in the graphics card memory. Fortunately, while two 3D textures are used to render a few frames, it is possible to upload the next required texture. This pipe process requires the storage of only three $3 \mathrm{D}$ textures on the graphics hardware.

The described technique speed is highly dependant on the size of the image and on the number of rendered geometry proxy planes. Following timings have been computed on a mid range GPU (Nividia Geforce6600 with $128 \mathrm{MO}$ of RAM). For an image of $512 \times 512$ we reach 12 fps using 256 geometry proxy planes, using only 96 planes we reach $20 \mathrm{fps}$, and with 512 planes we obtain only $6 \mathrm{fps}$. As we try to interpolate the isosurface position between proxy planes, the rendering quality is not to dependant on their number. When too few planes are used some artifacts are visible on the edges of the objects: the user can see through thin portions of the volume.

\section{Conclusion}

We proposed here a model for generation of $3 D+$ time CT scan from a physically based approach providing information about the evolution of organs shape. A great interest of this approach is that the simulated displacement data could be calculated with any kind of physically based modelling. Moreover, the proposed visualisation tool provides the clinician with more facilities for treatment planning.

Initial numerical evaluation of the proposed prototype has shown a good behaviour of our algorithms. However, very serious clinical verification should be carried out before their integration in a treatment planning software platform.

The provided rendering facilities permit interactive time visualisation of the reconstructed 4D CT Scan, permitting the clinicians to study organs shape and poistion evolution in time.

\section{Acknowledgements}

We thank the ARC (the French Association for Cancer Research) for their financial support and all our partners: Léon Bérard Centre and ETOILE ${ }^{1}$ project (Espace de Traitement Oncologique par Ions Légers) for their support.

\footnotetext{
${ }^{1}$ http://ETOILE.univ-lyon 1.fr
} 


\section{References}

[1] Brian Cabral, Nancy Cam, and Jim Foran. Accelerated volume rendering and tomographic reconstruction using texture mapping hardware. In VVS '94: Proceedings of the 1994 symposium on Volume visualization, pages 91-98, New York, NY, USA, 1994. ACM Press.

[2] Markus Hadwiger, Christoph Berger, and Helwig Hauser. High-quality two-level volume rendering of segmented data sets on consumer graphics hardware. In VIS '03: Proceedings of the 14th IEEE Visualization 2003 (VIS'03), page 40, Washington, DC, USA, 2003. IEEE Computer Society.

[3] Markus Hadwiger, Ivan Viola, Thomas Theuß1, and Helwig Hauser. Fast and flexible high-quality texture filtering with tiled high-resolution filters. In $\mathrm{Vi}$ sion, Modeling and Visualization 2002, pages 155162. Akademische Verlagsgesellschaft Aka GmbH, Berlin, November 2002.

[4] JD Humphrey. A possible role of the pleura in lung mechanics. J Biomech, 20(8):773-777, 1987.

[5] P J Keall, G Starkschall, H Shukla, K M Forster, V Ortiz, C W Stevens, S S Vedam, R George, T Guerrero, and R Mohan. Acquiring 4d thoracic ct scans using a multislice helical method. Physics in Medicine and Biology, 49(10):2053-2067, 2004.

[6] Thomas Klein, Simon Stegmaier, and Thomas Ertl. Hardware-accelerated reconstruction of polygonal isosurface representations on unstructured grids. In Computer Graphics and Applications, 12th Pacific Conference on (PG'04), pages 186-195, 2004.

[7] Marc Levoy. Display of surfaces from volume data. IEEE Computer Graphics \& Applications, 8(3):2937, May 1988.

[8] Adriano Lopes and Ken Brodlie. Interactive approaches to contouring and isosurfacing for geovisualization. In Exploring Geovisualization, pages 345361. Elsevier, 2005.

[9] N. Milickovic, D. Baltas, S. Giannouli, M. Lahanas, and N. Zamboglou. CT imaging based digitally reconstructed radiographs and their application in brachytherapy. Physics in Medicine and Biology, 45:2787-2800, 2000.

[10] Marylin L. Moy and Stephen H. Loring. Compliance. Seminar in respiratory and critical care medecine, 19(4):349-359, 1998.
[11] E. Rietzel, GT. Chen, NC. Choi, and CG. Willet. Four-dimensional image-based treatment planning: Target volume segmentation and dose calculation in the presence of respiratory motion. International Journal of Radiation Oncology Biology Physics, 61(5):1535-50, April 2005.

[12] S. Rit, D. Sarrut, and C. Ginestet. Respiratory signal extraction for $4 \mathrm{~d} c t$ imaging of the thorax from cone-beam ct projections. In MICCAI, pages 556563, 2005.

[13] P-F. Villard, M. Beuve, B. Shariat, V. Baudet, and F. Jaillet. Simulation of lung behaviour with finite elements : Influence of bio-mechanical parameters. IEEE, Mediviz, Conference on Information Visualization, pages 9-14, 2005. 